\title{
CULTURA E IDENTIDADE NA POLÍTICA PÚBLICA MUNICIPAL DE PARAGOMINAS - PARÁ ${ }^{1}$
}

\section{Culture and Identity in Municipal Policy on Paragominas - Pará}

\author{
Maria Aparecida Luciano \\ Maria José de Souza Barbosa ${ }^{3}$ \\ Walery Costa Reis ${ }^{4}$ \\ Farid Eid $^{5}$
}

Samuel Carvalho de Aragão ${ }^{6}$

\begin{abstract}
Resumo: O artigo apresenta uma discussão sobre o processo de construção da política municipal de cultura no município de Paragominas, estado do Pará, como estratégia de inclusão social de diferentes identidades. Trata-se de um debate necessário à reflexão sobre a implementação de ações de política pública que tem por princípio a participação democrática sob relação dialógica entre poder público e sociedade civil. Para se delinear esse debate percebeu-se a importância da escuta dos atores sociais: dança, audiovisual, multimídia, música, cultura popular, teatro, patrimônio material e imaterial, literatura, mídias contemporâneas, design, arte digital, artes visuais, arquitetura, respeitando-se a identidade cultural desses diversos atores, a fim de se valorizar a multiplicidade de manifestações que abrangem o patrimônio cultural existente no município. Objetivou-se com este artigo mostrar as diferentes dimensões da cultura enquanto reflexos da sociedade, sua dinâmica na construção das identidades dos diferentes grupos que constroem a sociedade paragominense em suas especificidades. Apoiou-se no debate teórico referenciado em Stuart Hall e Nestor Garcia
\end{abstract}

\footnotetext{
1 Artigo produzido com base na Dissertação desenvolvida no âmbito do Programa de Pós-Graduação em Desenvolvimento Rural e Gestão de Empreendimentos Agroalimentares, do Instituto Federal de Educação, Ciência e Tecnologia do Pará (IFPA).

${ }^{2}$ Mestre em Desenvolvimento Rural e Gestão de Empreendimentos Agroalimentares, pelo Instituto Federal de Educação, Ciência e Tecnologia do Pará (IFPA), professora de Língua Portuguesa e Secretária Municipal de Cultura, Turismo, Desporto e Lazer do Município de Paragominas - PA. Orcid: https://orcid.org/0000-0002-06000824. E-mail: cida_luciano@hotmail.com.
}

\footnotetext{
3 Doutora em Serviço Social, pela Universidade Federal do Rio de Janeiro (UFRJ), com pós-doutorado em Geografia, pela Universidade de Alicante - UA, professora do Programa Pós-Graduação em Desenvolvimento Rural e Gestão de Empreendimentos Agroalimentares do Instituto Federal de Educação, Ciência e Tecnologia do Pará (IFPA). Orcid: https://orcid.org/0000-0002-7644-8923. E-mail: majose@ufpa.br.

${ }^{4}$ Doutor em Administração pela Universidade Federal de Pernambuco (UFPE), professor do Instituto Federal de Educação, Ciência e Tecnologia do Pará (IFPA). E-mail: walery.reis@ifpa.edu.br.

${ }^{5}$ Doutor em Economia e Gestão, pela Université Picardie Jules Verne, professor do Programa de Pós-Graduação em Desenvolvimento Rural e Gestão de Empreendimentos Agroalimentares, do Instituto Federal de Educação, Ciência e Tecnologia do Pará (IFPA). Orcid: https://orcid.org/0000-0002-5491-4516. E-mail: fe9998@ gmail.com.

${ }^{6}$ Doutor em Medicina Veterinária pela Universidade Estadual Paulista (UNESP), professor do Instituto Federal de Educação, Ciência e Tecnologia do Pará (IFPA). E-mail: samuelaragao@mec.gov.br.
} 


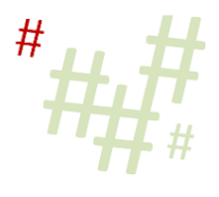

Canclini, os quais possibilitaram a ampliação do conceito de cultura ao abordarem diferentes dimensões da vida social de um povo. Conclui-se com a perspectiva de abertura para uma concepção democrática da política cultural, enquanto necessária ao desenvolvimento socioeconômico e cultural.

Palavras-chave: Política Pública. Cultura. Desenvolvimento Municipal.

\begin{abstract}
The article presents a conceptualization of culture in the anthropological and sociological dimensions, aiming to support the structuring of a cultural policy in the city of Paragominas, state of Pará, as a strategy for social transformation. This debate is necessary for reflection on the implementation of public policy actions that have democratic participation as their principle, in a cohesive manner between the government and civil society. In order to delineate this debate, the importance of open dialogue and listening to the expressions of social actors: dance, audiovisual, multimedia, music, popular culture, theater, material and immaterial heritage, literature, contemporary media, design, digital art, visual arts, architecture, respecting the cultural identity of all actors were perceived, in order to value the diversity of manifestations that encompass the existing cultural heritage in the municipality. This article aimed to show the different dimensions of culture as a reflection of society, its dynamics in the construction of identities of the different groups that build societies in their specificities. Methodologically, a theoretical debate was made with the first references authors Stuart Hall and Nestor Garcia Canclini for broadening the concept of culture to encompass all the expressions of the social life of a people. It concludes with the prospect of openness to a democratic conception of cultural policy as necessary for social development.
\end{abstract}

Keywords: Public Policy. Culture. City Development.

\title{
1 Introdução
}

O artigo tem como objetivo discutir o processo de expansão da política municipal de cultura às periferias, zona periurbana e rural de Paragominas, no Nordeste do Pará. Trata-se, portanto, de um processo de ampliação do conceito de cultura. Mostra-se, a partir da compreensão de Hall (2003, 2002, 1997, 1996) e Canclini (2005, 2001, 1987) diferentes dimensões da cultura enquanto reflexos da sociedade, sua dinâmica de construção por identidades impressas por diferentes grupos sociais que, em suas especificidades, metamorfoseiam-se e produzem inovações na totalidade das expressões da vida social.

O universo da cultura torna-se o fio condutor para se refletir e redimensionar a extensão da política pública de cultura em Paragominas. Por essa razão, os autores referenciados possibilitaram a ampliação do conceito de cultura, favorecendo, assim, uma visão estratégica, consonante com as diversas vozes que ecoam das múltiplas identidades que se modificam na interação com outras e pela efemeridade do tempo que se transforma nessa inter-relação.

Para melhor compreensão, dividiu-se o artigo em dois grandes eixos: no primeiro apresenta-se uma concepção de cultura construída no diálogo aberto entre diferentes identidades locais, a fim de escutar os atores, suas expressões e linguagens artísticas, para valorizar as manifestações que constituem o patrimônio cultural existente no município, fenômeno ocasionado face à dinâmica dos movimentos sócio históricos e econômico-culturais que se transmutam constantemente. 
No segundo eixo, procura-se vislumbrar os passos do processo de extensão da Secretaria Municipal de Cultura (SECULT), a partir de uma experiência piloto na Colônia do Uraim, comunidade rural localizada a $11 \mathrm{~km}$ da sede do município, cuja população é de 2.000 habitantes, distribuídos em 400 famílias. Essa experiência foi realizada no âmbito do estágio obrigatório do Programa de Pós-graduação em Desenvolvimento Rural e Gestão de Empreendimentos Agroalimentares, Mestrado profissional, do Instituto Federal de Educação, Ciência e Tecnologia do Pará (IFPA) - Campus Castanhal, em que há a necessidade de uma inserção efetiva do mestrando para contribuir com a sociedade.

Por meio desse estágio, realizou-se um inventário das manifestações culturais do município, momento em que se observou a inexistência de atividades culturais na zona rural. Destacou-se a Colônia do Uraim onde havia demandas apresentadas por lideranças locais ao prefeito municipal, quanto a realização de ações para a inclusão social de crianças e adolescentes. Com base nesses dois documentos, estabeleceu-se um espaço de encontro na Escola Municipal de Ensino Fundamental Comunitária do Uraim, quando se definiu pelo desenvolvimento de um projeto de extensão da SECULT, denominado Uraim Cultural.

O público prioritário dessa experiência foi aquele em situação de evasão escolar, apresentando histórico de mau relacionamento familiar e delinquência juvenil (vandalismo ou uso de drogas), portanto, jovens que se encontravam fora da escola. A proposta teve como finalidade promover a inclusão social de crianças e adolescentes, por meio de práticas pedagógicas culturais e esportivas, respeitando a diversidade e a identidade local, para estimular competências e habilidades artísticas voltadas ao exercício da cidadania; bem como, para contribuir na reinserção de jovens ao ambiente escolar e comunitário.

\section{Cultura e identidade na formulação da política pública municipal}

Articulou-se o pensamento explicitado nos estudos culturais de Hall (2001) e o debate de Canclini (1995) para entender as práticas culturais em busca de reconhecer e legitimar as expressões e manifestações dos diversos segmentos populacionais, na medida em que se visava promover meios de inclusão sociocultural dos sujeitos sociais implicados nas ações da política pública municipal de cultura. Era preciso encontrar elos que pudessem vislumbrar identidades em uma sociedade de desenvolvimento tardio e em mutações, gerados pelos processos de globalização.

A cultura é uma forma de regulação normativa e, nesse aspecto, busca dar sentido às condutas e práticas humanas, direcionando propósitos, fins e intenções, por meio de valores comuns. Nesse sentido, segmenta grupos e indivíduos segundo padrões impostos, pois “"...] as ações sociais são significativas tanto para aqueles que as praticam quanto para os que as observam: não em si mesmas, mas em razão dos muitos e variados sistemas de significado [...]" (HALL, 1997, p. 1).

Os seres humanos constroem e dinamizam a sociedade com suas práticas sociais oriundas de conhecimentos pretéritos, crenças, hábitos, valores, costumes próprios, os quais compõem um mosaico de ações em um determinado ambiente histórico (homem-natureza) que é, ao mesmo tempo, social, econômico e cultural.

Os estudos culturais, nesse sentido, possibilitam entender hábitos e manifestações grupais, particularidades e individualidades. Eles levam à percepção de valorização ou mesmo rejeição de determinados grupos sociais. Por meio da análise cultural pode-se observar e interpretar ações que envolvem os indivíduos em contextos locais. Hall (1997, p.6) considera que a cultura se insere nas "vozes e imagens incorpóreas que nos interpelam das telas, nos 
postos de gasolina. Ela é um elemento chave no modo como o meio ambiente doméstico é atrelado, pelo consumo, às tendências e modas mundiais".

A análise cultural se volta aos produtos, usos, instrumentos, expressões, símbolos, valores, focando em elementos objetivos e na subjetividade dos sujeitos e seus grupos sociais. Identifica narrativas em um contexto prenhe de significados, os quais se metamorfoseiam nos processos de interação e repulsão de um mundo em constantes transformações, onde "[...] velhas certezas e hierarquias da identidade britânica [e global] têm sido postas em questão [...] um alargamento do campo das identidades e uma proliferação de novas posições - de identidade-, juntamente com o aumento de polarização entre ela [...] (HALL, 2002, p.84).

Na sociedade contemporânea na globalização há a possibilidade do fortalecimento de identidades locais, de forma contraditória, face às dimensões variadas que traduzem determinados espaços sob uma profusão de novos traços, gerando culturas híbridas, as quais surgem da interação, absorção e afirmação de traços ancestrais, que passam a gerar novas dimensões societais.

Neste campo, Canclini (1987) trabalhou o conceito de políticas culturais como algo que deve ser constituído de maneira participativa, compartilhada entre poder público e sociedade civil em geral, tendo em vista que a função da política cultural é de satisfazer as necessidades culturais da população. Por essa razão, a política cultural precisa se constituir por um conjunto de intervenções, em que as instituições civis, o estado e grupos organizados se orientam na valorização/promoção de manifestações simbólicas.

Nessa acepção de política cultural democrática, os indivíduos não podem ser tomados apenas como consumidores, mas como cidadãos imersos nas tensões políticas antagônicas de classes e de grupos. Sem o exercício da cidadania cultural não há uma verdadeira democracia cultural. A política cultural, nesses termos, não deve se restringir à difusão de expressões e manifestações culturais hegemônicas, mas promover o desenvolvimento de todas que representem os diferentes grupos identitários que compõem uma sociedade (CANCLINI, 1987).

Nesta perspectiva, coloca-se a necessidade de democratização das políticas culturais, a fim de assegurar que haja ampliação na distribuição dos bens culturais materiais e imateriais entre a população, criar condições de acesso desta às instituições e aos espaços públicos culturais, permitindo que o cidadão possua maior consciência crítica e estética frente às manifestações culturais diversas.

As estratégias de uma política cultural são estabelecidas pela compreensão e abrangência da definição de cultura. Desta forma, a política cultural precisa ter clareza das diferentes manifestações e expressões culturais, em suas dimensões e universo, distinguindo-a no plano do cotidiano, onde ocorre a dinâmica da vida social. Para isso, é necessário mobilizar várias estratégias para dinamizar as características estruturais (materiais e simbólicas), condição de valorização dos sujeitos.

$\mathrm{Na}$ formulação de políticas municipais de cultura, há a necessidade de se pensar e identificar objetivos e metas a serem traçadas, para estabelecer ações que favoreçam as manifestações e expressões dos atores diversos, em seu caráter privado e público. Neste aspecto particular, a interação social precisa ser entendida como elemento essencial na elaboração de políticas públicas culturais. Os modos de pensar e sentir determinam valores, que por sua vez marcam identidades e diferenças, além de estabelecer rotinas e construir expressões típicas de uma dada região. 
É na sociedade que os indivíduos produzem seus mundos particulares e coletivos com seus tipos diversos de símbolos e artefatos, dando sentido à existência individual e social. A cultura é essencialmente constituída pela relação entre homens e coisas, bens tangíveis e intangíveis.

Botelho (2001) chama atenção para as dimensões antropológica e sociológica da cultura, mas pode-se agregar as dimensões econômica, política e social. Isto porque nessas diferentes esferas da vida social encontram-se elementos que marcam e identificam uma determinada sociedade.

$\mathrm{Na}$ formulação de políticas públicas culturais é necessário por em movimento as expressões e manifestações que marcam e/ou caracterizam uma sociedade. Nesse sentido, a participação dos atores sociais torna-se um elemento importante no compartilhamento, na divulgação e na difusão. E à política pública cabe criar espaços para que estas sejam visibilizadas enquanto auto reconhecimento.

Na formulação de políticas públicas culturais exigem-se profissionais capazes de fazer valer as necessidades de expressão, valorização e reconhecimento, por meios institucionais, para dar voz aos sujeitos que constituem o universo cultural em suas diferentes dimensões e expressões do público, capazes de atender às demandas dos cidadãos.

A política cultural precisa englobar uma multiplicidade de expressões, como resultado da ação do conjunto da sociedade, em razão da diversidade de expressões que se constituem como a memória de um povo, na construção de mundo, nos processos de relação do homem com a natureza e com os demais homens.

A interação e o intercâmbio, a troca de bens culturais materiais e simbólicos favorecem novas possibilidades ao potencializar ações e relações entre os povos. "As ações nesse sentido devem partir de um pacto com a sociedade civil, aquela que demanda políticas públicas" (LEITÃO, 2009, p.9). A abrangência das ações governamentais depende da própria concepção de cultura, da definição de que expressões valorizar, ou mesmo obscurecer, ao estabelecer critérios de apoio e investimentos, ou seja, dos parâmetros que permitem a delimitação de suas estratégias na divulgação e difusão por meio das políticas culturais.

No Brasil, com suas dimensões continentais, há uma variedade constitutiva de manifestações que pode ser visualizada por meio de uma rica produção cultural. A diversidade sociocultural do país expressa variações derivadas de seus diversos grupo étnico-raciais, sob espaços onde transitam práticas sociais de seu povo multirracial e multicultural, que precisam ser incorporados e expandidos em termos de difusão de suas identidades em constante mutação.

A ampliação da visão e dos espaços de compreensão dos modos de vida dos diferentes grupos sociais é observada em Gil (2003), quando pensa: "Nossa tarefa comum é a de formular e executar políticas públicas de cultura, articuladas e democráticas, que promovam a inclusão social e o desenvolvimento econômico, e consagrem a pluralidade que nos singulariza entre as nações, e que singulariza, dentro da nação, as comunidades que a compõem".

Nessa compreensão de política cultural vê-se a dinâmica das transformações que movem a sociedade, em suas diferentes dimensões, desde a fala, hábitos e costumes que se metamorfoseiam, mas de traços que permanecem arraigados, constitutivos de similitudes e contrastes, nos contextos das relações familiares e de vizinhança, mas também da sociabilidade, num sentido amplo.

A cultura enquanto política pública salta do cotidiano para se constituir no plano de uma ação planejada e organizada, a fim de alcançar tipos específicos de público. Para isso ela 
depende de um conjunto de fatores que vão desde a decisão política ao estabelecimento de um aparato técnico-burocrático em condições de garantir a estruturação de formas de expressão e manifestações, onde estas possam ser divulgadas e difundidas. A política cultural exige ambientes propícios, enquanto canais de promoção das diferentes expressões, muitas vezes restritos em face aos recursos financeiros e orçamentários destinados a seus meios específicos de expressão.

Nesse sentido, as políticas públicas precisam fortalecer laços, alianças e negociações, para encontrar meios de coesão, que favorecem a todos. A mobilização das capacidades criativas, sob um universo de ações e atividades torna-se uma estratégia importante para construir mecanismos de gestão de diferentes naturezas artísticas, a partir de programações, em meio a uma complexidade difícil de se capturar em sua integralidade, geralmente, constituída por foco de atenção das políticas culturais.

O caráter público das políticas governamentais, com orçamentos restritos, deixa de lado uma infinidade de expressões, isto é, criando um universo próprio de atenção ao estruturar ações institucionais em sistemas organizados da ação cultural. As políticas públicas culturais criam, assim, determinados circuitos de produção, promoção, meios de divulgação para determinados públicos, ao implementarem programas e projetos de incentivos e estímulos em termos de financiamento.

Nesse contexto, a democratização passa a ser um desafio, na medida em que os recursos para essas iniciativas são do conjunto da sociedade. As ações de políticas públicas culturais enquanto estimuladoras dos diversos meios de produção cultural pode se constituir como um importante veículo de democratização de "bens simbólicos", além dos materiais.

O ordenamento público estrutural das expressões e atividades culturais precisa mostrar a capacidade de estabelecer, em termos de uma política pública, a democratização das mais diversas expressões e não se restringir a manifestação privilegiada. A ação sociocultural é, em sua essência, de caráter micro que tem maior proximidade na instância administrativa municipal.

A ação de política pública municipal de cultura exige planejamento face a escassez de recursos, o que reduz sua capacidade de absorção da multidimensionalidade das expressões. $\mathrm{E}$ quando há interferências externas (por interesses alheios), ela se restringe a determinadas áreas artísticas e tem a necessidade de buscar resultados relativamente previsíveis.

Neste circuito da política pública municipal, trata-se de expressão artística de modo restrito, onde o orçamento e as opções de financiamento são critério da gestão, nem sempre exercida de modo democrático. $\mathrm{O}$ aparato municipal para propiciar o acesso às diversas linguagens precisa ser democrático, pois quando isso não ocorre ou mesmo se o seu exercício é descompromissado, não repercute na dimensão pública do receptor ou difusor de bens culturais.

A institucionalização de um certo universo cultural, com suas próprias características, certamente torna-se um campo privilegiado das políticas culturais, na medida em que seleciona e promove determinados segmentos e passa a dar visibilidade social às expressões destacadas.

O inventário do patrimônio cultural, quando há o diálogo direto com os atores sociais, possibilita a realização de diagnósticos da realidade cultural, no âmbito municipal, de modo a contribuir para a inclusão de expressões culturais, estimar recursos e metas em curto, médio e longo prazos. As políticas culturais, isoladamente, não conseguem atingir o plano do cotidiano multivariado (BOTELHO, 2015). 
Para ampliação da ação de política pública, é preciso criar espaços de debates voltados ao estabelecimento de compromissos entre os diferentes atores sociais, para organizar agendas quanto às ações, programas e projetos a serem realizados. Os gestores públicos precisam de habilidades específicas na construção de estratégias de inserção dos produtores culturais, diante dos desafios e disputas de poder junto aos demais setores da máquina governamental. É necessário superar concepções departamentalizadas. A intersetorialidade, como estratégia de governo, favorece pontos de convergências entre as diferentes dimensões da vida cultural, para agregar expressões do esporte, lazer, dança, música, teatro, artesanato, geração de renda, junto à assistência social, à educação, à agricultura etc.

Para Botelho (2001), o setor da cultura deve funcionar como articulador de programas conjuntos, pois seu compromisso de governo precisa encontrar-se com o conjunto de atores individuais e coletivos, na criação de condições para a interação social dos diferentes segmentos sociais. Isso significa que a cultura, enquanto tal, em sentido lato, exige a articulação política efetiva de todas as áreas da administração, para alcançar o plano do cotidiano. Neste aspecto, o compromisso de todas as instâncias governamentais é essencial, pelo fato de a política cultural ter caráter interdisciplinar e ultrapassar fronteiras departamentalizadas.

A Secretaria Municipal de Cultura depende de articulação do gestor, junto ao Poder Executivo, para construir um planejamento da política cultural pública, capaz de captar a sua multifuncionalidade, na medida em que se trata de qualidade de vida, não podendo ser vista como algo secundário e acessório, mas um mecanismo de inclusão cidadã.

Em Botelho (2001) vê-se que as políticas culturais, nas esferas administrativas, acabam distanciando-se dos grupos sociais, na medida em que as estruturas burocráticas segmentam a vida efetiva do cidadão, embora legitime política, econômica e socialmente os grupos que as promovem.

\section{Política municipal de cultura em Paragominas}

Os estudos culturais de Hall (1997), complementados pela concepção de democratização cultural de Canclini (1987), levou-nos a aproximar as lentes sobre a realidade do município de Paragominas ${ }^{7}$. Procurou-se entender como era possível ampliar a política pública municipal a fim de abranger as múltiplas identidades constituídas, em um município que já traz em seu próprio nome a diversidade imanente aos estados brasileiros (Pará, Goiás e Minas Gerais) e, mais recente, maranhenses, paranaenses, gaúchos, goianos, mato-grossenses, além de outras identidades culturais.

\footnotetext{
${ }^{7}$ O processo de ocupação da área que mais tarde daria origem ao município de Paragominas está relacionado ao povoamento do Estado do Pará, na década de 50, a partir da abertura de Rodovias e Projetos de Colonização. Foi efetivada com a presença de camponeses, que foram os pioneiros na região, antes da construção da rodovia BelémBrasília, seguidos pelas primeiras companhias colonizadoras: Colonizadora Belém-Brasília, Colonizadora Marajoara e Cidade Marajoara, que não obtiveram êxito. Mais tarde, o governo federal divulgou a instalação de uma colônia federal na região, que nunca chegou a se estabelecer, bem como os planos estaduais para a formação de duas colônias naquele território. Registra-se, também, que antes mesmo da chegada dos camponeses, com autorização do Governo do Estado, especuladores de Goiás haviam penetrado na floresta, ao longo do rio Capim, com o objetivo de efetuar levantamentos e titular terras para compradores de Uberaba e Itumbiara, em Minas Gerais. Posteriormente, a proximidade da rodovia BR-010 (Belém-Brasília), provocou uma grande procura pelas terras entre proprietários de Minas Gerais e Espírito Santo, além de companhias de especulação de terras de São Paulo, ao mesmo tempo em que camponeses penetravam na região, com o objetivo de enfrentar a competição com os "grileiros", que emitiam títulos falsos e os asseguravam, através do uso da força (PARÁ, 2011, p.6).
} 
No evolver da história municipal, as diferentes identidades modificam-se à medida que vão estabelecendo processos de interculturalidade, sob um ambiente de transformações das paisagens naturais e sociais, corroborando as ideias de uma democracia cultural (CANCLINI, 1987).

Assim, elaborou-se um inventário ${ }^{8}$ das expressões culturais existentes no município de Paragominas, como forma de entender as diferentes manifestações culturais em um território múltiplo para pensar, estrategicamente, a formatação de uma política em que as diferentes vozes fossem representadas.

O inventário cultural, desta forma, possibilitou-nos o conhecimento de uma diversidade de expressões culturais, como bandas e fanfarras escolares e de associações, grupos de quadrilhas juninas, grupos de capoeira, danças urbanas (tecno-brega, funk, hip-hop), danças regionais, uma riqueza de manifestações nos bairros periféricos do município e na zona rural, que não eram, até então, incluídos, de forma sistematizada, na programação da Secretaria Municipal de Cultura de Paragominas.

Com base nesse banco de dados, selecionou-se a Colônia do Uraim, caracterizada em sua origem por um projeto de colonização fracassado de 1962, cuja existência é anterior à própria emancipação do município de Paragominas, datada de 1965. Constituída, hoje, por 400 famílias assentadas, trabalhadores da agricultura familiar; a Colônia possui poucos equipamentos públicos (duas escolas que atendem da creche ao $9^{\circ}$ ano e um posto de saúde) e tem sérios problemas de trafegabilidade, dificultando o acesso dos moradores à sede do município, principalmente para o escoamento da produção.

A Colônia do Uraim tornou-se um território estigmatizado na municipalidade, devido a situações de vandalismo, uso de entorpecentes, criminalidade (furtos, roubos, homicídios), assim como pela falta de oportunidade de emprego e geração de renda para a juventude. Por esses condicionantes, foi selecionada para o estágio vinculado ao Mestrado Profissional. Havia um documento de lideranças locais ao gestor municipal, solicitando ações que pudessem incidir sobre os altos índices de violência associada à falta de oportunidades para a juventude local.

Desse movimento, criou-se um espaço de diálogos diretos, através de encontros de gestores públicos municipais com atores sociais, originando a formulação do projeto de extensão da SECULT, denominado Uraim Cultural. Uma estratégia de enfrentamento dos problemas, por meio de inclusão sociocultural.

A compreensão dos processos de formação sócio histórica do município foi essencial para a inclusão desses atores nas ações de democratização da cultura, abrindo espaço para diferentes dimensões da vida social dos grupos locais, com suas expressões e manifestações culturais que passaram a fazer parte da política municipal de cultura.

Jovens produtores de cultura, em suas comunidades rurais, passaram a apresentar experiências e vivências socioculturais sob um processo dinâmico e participativo. Ali, os jovens mostram-se empoderados e valorizados. No entanto, para a manutenção desses grupos, no decurso de anos, esses coletivos tem promovido rifas, torneios de futebol, feijoadas e bazares, para arrecadar fundos necessários à aquisição de indumentárias, adereços, custeio de viagens para participação em eventos em municípios vizinhos.

\footnotetext{
${ }^{8}$ De acordo com a concepção do IPHAN, os inventários são realizados como mecanismo que visam identificar as manifestações culturais de natureza material e imaterial, existentes em um determinado território, para efeito de preservação. Constitui um banco de dados das diferentes expressões como forma de valorização e, ao mesmo, subsídio para ações de planejamento, pesquisa, conhecimento patrimonial.
} 
A participação popular, na escuta das diversas vozes, com suas demandas e potencialidades, favoreceu a construção de uma ação de política pública municipal em Paragominas, ancorada nas demandas locais.

A experiência do projeto Uraim Cultural levou a Secretaria Municipal de Cultura a criar uma agenda pública em que artistas individuais ou em grupo (jovens do segmento de bandas e fanfarras, do segmento Folclore, Festa Junina e concurso de quadrilhas) e ainda com diferentes expressões da música (cantores, compositores e bandas locais) tiveram suas participações asseguradas, inclusive, com recebimento de cachês, em pelo menos um evento realizado pela Prefeitura.

Com essa ação pode-se promover a democratização da política pública municipal de cultura, de forma descentralizada, compartilhada e participativa, dando voz aos atores, especialmente, aos jovens de baixa renda, do campo e da periferia, cujo acesso aos bens culturais é limitado, dada à inexistência de equipamentos públicos adequados, como teatros, salas de cinema, centros de dança, existentes somente na sede da municipalidade.

Nesse sentido, a Colônia do Uraim tornou-se um espaço privilegiado, onde se pode escutar os atores dessa localidade (crianças, jovens e pais) que explicitaram suas preferências por determinadas atividades culturais e do desporto. Essa abertura à participação popular através do diálogo, favoreceu a implementação do projeto Uraim Cultural, nessa comunidade.

A democratização dos espaços públicos, para o exercício das expressões culturais, não se constitui em uma tarefa fácil, é necessário um esforço e compromisso de gestores de diferentes áreas, na medida em que se constitui como uma ação multidimensional de caráter intersetorial. Os jovens, muitas vezes, não conseguiam permissão para uso de determinados locais, como as quadras das escolas, para realizar seus ensaios, fato esse resolvido com diálogo entre a Secretaria de cultura que passou a mediar as relações entre os atores locais, gestores da educação e os jovens produtores culturais e artistas, tendo como referência o projeto de extensão cultural da SECULT, na Colônia do Uraim, onde a gestão da Escola de Ensino Fundamental cede o espaço para a realização das atividades do projeto Uraim Cultural.

Nesse espaço procurou-se envolver diversas linguagens, pois a perspectiva maior era aplicar os conceitos de identidades variadas, culturas híbridas e democratização cultural. Neste aspecto, o município de Paragominas tem potencializado diferentes identidades, em suas formas de sentir, pensar e agir, um manancial de cultura popular, com suas diferentes práticas sociais, expressões e linguagens folclóricas (tradicionais) e modernas (danças de rua, por exemplo).

Neste aspecto, o conceito de identidade acionado não supõe qualquer conotação de homogeneidade, pelo contrário, reforça a heterogeneidade, a diversidade cultural e a existência de múltiplas juventudes. Em Paragominas, percebe-se a mutação dessas identidades efêmeras, capazes de respostas ágeis, por vezes, surpreendentes e comprometidas, mas também elementos tradicionais, como as bandas e fanfarras que permanecem como expressões de um tempo que traz o passado, mas que se comunica com o presente, em sua riqueza que transpõe bordas entre o novo e o velho, no simbolismo popular.

\section{Considerações finais}

O projeto Uraim Cultural, de extensão da Secretaria Municipal de Cultura de Paragominas, tornou-se um mecanismo de democratização da política pública municipal, ao acionar diferentes expressões culturais imanentes ao movimento de formação socioeconômica 
e cultural, inclusive, absorvendo novos sujeitos e suas identidades oriundas do movimento migratório recente, que se mostra no campo e na cidade.

A ação de política cultural via Uraim Cultural definiu estratégias diversificadas de articulação entre diferentes variáveis que pudessem incluir as formas de vida da localidade, sem perder de vista a dinâmica de globalização da sociedade, cada vez mais influenciada pelos meios de comunicação e linguagens diversificadas (em tempo real e virtual), particularmente quando associada aos novos meios midiáticos e computacionais, na produção e reprodução da vida (âmbito doméstico) e no público face ao pertencimento social.

A identidade, pode-se dizer, tornou-se um elemento essencial na formulação de ações de políticas públicas voltadas à valorização das diversas manifestações culturais, em meio a um movimento mutacional constitutivo da própria vida social de Paragominas.

Nesse espaço público, gera-se uma profusão de culturas que se comunicam, se aproximam e, em alguns casos, se chocam tensionadas por processos híbridos, de fortalecimento ou de disputas na produção e emersão de novas identidades, as quais precisam ser capturadas na formulação das políticas públicas municipais de cultura.

Assim, privilegiar a Colônia do Uraim foi uma estratégia de democratização da cultura para expandir os espaços de expressões e manifestações de grupos sociais até então marginalizados, na medida em que as ações de políticas públicas municipais de cultura e esporte, em Paragominas, eram restritas ao distrito sede, ou seja, à cidade.

Desta forma, as contribuições teóricas de Hall e Canclini possibilitaram a compreensão das diferentes identidades e favoreceram o entendimento quanto a necessidade de promover novos espaços político-culturais, com suas diferentes dimensões que passaram a ser incorporadas na política pública municipal de Paragominas, formadas a partir de diversas representações e significações também híbridas, movidas pelo sentimento de pertencimento e de diferença, como um processo enunciativo proveniente de várias vozes e histórias ressonantes.

No projeto de extensão Uraim Cultural há um movimento contínuo de busca de interações com os sujeitos que criam, em seus espaços, "produtos" próprios em inter-relações internas e externas às comunidades locais, em um contexto permeado por dinâmicas regionais, nacional e global, favorecendo mutações e transformações sócio históricas de constituição municipal.

$\mathrm{Na}$ formulação de políticas públicas municipais, portanto, é necessário entender a dinâmica da vida social, não somente na valorização de campos específicos, mas repercutir em ganhos sociais, econômicos, políticos fundamentais, particularmente, como um bem coletivo condutor de desenvolvimento com inclusão social.

A identidade em sua multidimensionalidade passou a ser compreendida inserida nas ações de políticas públicas de cultura. Uma lição apreendida nesse espaço público criado no diálogo entre atores sociais e a Secretaria Municipal de Cultura de Paragominas, mesmo com dificuldades decorrentes, seja do acesso, seja dos distanciamentos, seja dos recursos. Uma experiência formada por representações e significações híbridas, em um processo enunciativo de várias vozes e histórias ressonantes, constituídas por identidades que ocupam significados expressivos de seus tempos de interlocução com as diferentes esferas da sociedade e com grupos sociais diferenciados. 


\section{Referências}

BOTELHO, Isaura. Dimensões da cultura e políticas públicas. São Paulo em Perspectiva, São Paulo, v. 15, n. 2, p.73-83, 2001. Disponível em https://www.scielo.br/scielo.php?script=sci_arttext\&pid=S0102-88392001000200011. Acesso em: 14 fev., 2020.

BRASIL. Instituto do Patrimônio Histórico e Artístico Nacional - IPHAN. http://portal.iphan.gov.br/pagina/detalhes/421. Acessado em 13/10/2020.

CANCLINI, Néstor Garcia. Consumidores \& cidadãos: Conflitos multiculturais da globalização. Rio de Janeiro: UFRJ, 1995.

CANCLINI, Néstor Garcia. Cultura, política y sociedad: Perspectivas latinoamericanas. Buenos Aires: Consejo Latinoamericano de Ciencias Sociales, 2005.

CANCLINI, Néstor Garcia. Diferentes, desiguais e desconectados: Mapas da interculturalidade. Rio de Janeiro: UFRJ, 2006.

CANCLINI, Néstor Garcia. La Globalización Imaginada. Buenos Aires: Paidós, 2001.

CANCLINI, Néstor Garcia. Politicas Culturales en America Latina. Cidade do México: Editorial Grijalbo, 1987.

GIL, Gilberto. Discursos do Ministro no Encontro dos Secretários de Cultura. Disponível em: www.cultura.gov.br. Acesso em: 14 fev., 2020.

HALL, Stuart. A centralidade da cultura: notas sobre as revoluções culturais do nosso tempo. Educação \& Realidade, Porto Alegre, v. 22, n. 2, p. 15-46, 1997.

HALL, Stuart. A identidade cultural na pós-modernidade. Tradução Tomaz Tadeu da Silva, Guaracira Lopes Louro. São Paulo: DP\&A, 2001.

HALL. Identidade cultural e diáspora. Revista do Patrimônio Histórico e Artístico Nacional, Rio de Janeiro, p. 68-75, 1996.

HALL, Stuart. Da Diáspora: identidades e mediações culturais. Belo Horizonte: UFMG, 2003.

HALL, Stuart. Modernidade e Identidade. Rio de Janeiro: Zahar, 2002.

LEITÃO, Cláudia. Cultura e Municipalização: Cultura é o que? Salvador: Fundação Pedro Calmon, v. 03, 2009.

LUCIANO, Maria Aparecida. Transformações sociais na trajetória da política municipal pública cultural de Paragominas - Pará. Castanhal: IFPA, 2019. (Dissertação de Mestrado)

MINISTÉRIO DA CULTURA. Cultura Viva: Programa Nacional de Cultura, Educação e Cidadania. Brasília: MINC, 2005. 
PARÁ. Instituto de Desenvolvimento Econômico, Social e Ambiental do Pará. Anuário Estatístico. Belém: IDESP, 2011.

PARAGOMINAS. Projeto de Extensão da Secretaria Municipal de Cultura de Paragominas: Uraim Cultural. Paragominas, 2018-2019. (Relatório Anual).

Recebido em maio de 2020.

Aprovado em novembro de 2020. 